\title{
Hybrid Key Duplication Hashing Techniques for IP Address Lookup
}

\author{
Rujiroj Tiengtavat, Wei-Ming Lin \\ Department of Electrical and Computer Engineering, The University of Texas at San Antonio San Antonio, USA \\ E-mail:weiming.lin@utsa.edu \\ Received February 9, 2011; revised March 26, 2011; accepted April 12, 2011
}

\begin{abstract}
In the past decade there has been an increasing need for designs to address the time and cost efficiency issues from various computer network applications such as general IP address lookup and specific network intrusion detection. Hashing techniques have been widely adopted for this purpose, among which XOR-operation-based hashing is one of most popular techniques due to its relatively small hash process delay. In most current commonly used XOR-hashing algorithms, each of the hash key bits is usually explicitly XORed only at most once in the hash process, which may limit the amount of potential randomness that can be introduced by the hashing process. In [1] a series of bit duplication techniques are proposed by systematically duplicating one row of key bits. This paper further looks into various ways in duplicating and re-using key bits to maximize randomness needed in the hashing process so as to enhance the overall performance further. Our simulation results show that, even with a slight increase in hardware requirement, a very significant reduction in the amount of hash collision can be obtained by the proposed technique.
\end{abstract}

Keywords: Hash Algorithm, IP Address Lookup, Intrusion Detection

\section{Introduction}

Fast address lookup or identification matching has become critical to the feasibility of many modern applications. In a general form, this problem involves a search process through a large database to find a record (or records) associated with a given key. One modern example is in that the routers in wide-area networks have to look through a large database, a routing table, for a forwarding link that matches the given destination address [2]. Another example that calls for imminent attention these days is in the area of internet security, in which intrusion detection demands rapid evaluation of client requests. In this, rules are established to allow the intrusion detection system to check for wrong-doing. There have been many designs developed for IP address lookup problem using a hash function. Hashing provides a way to search through a statistically smaller number of steps than a simple sequential straightforward search. Hashing in essence provides a process of mapping records (hash keys) between two regions, a domain space (the database) and a hash space [3]. An algorithm, known as a hashing function, issues a set of directives outlining the mapping of a hash key (or record) from the domain space into hash space by creating a corresponding hash value.

A complete survey and complexity analysis on IP address lookup algorithms has been provided in [4]. A performance comparison of traditional XOR folding, bit extraction, CRC-based hash functions is given in [5]. Although most of the hash functions, such as the simple XOR folding and bit extraction, are relatively inexpensive to implement in software and hardware, their performance tends to be far from desirable. CRC-based hash functions are proved to be excellent means but are more complex to compute. Some schemes are hardware based that achieve an improvement in IP look-up by maintaining a subset of routing table in a faster cache memory [6,7], while others are software based which improve their search performance mainly through efficient data structures [8,9]. Waldvogel et al. [10] proposed an address look-up scheme based on a binary search of hash table, requiring an extra update process in a look-up table. Other hashing algorithms have also been widely adopted to provide for the address look-up process [11-16]. All hashing algorithms inevitably suffer 
from unpredictable complexities involving conflicts among the data with the same hash result (hash collision). A search for matching a given query could end up with a sequential search through the number of maximal conflicts in the database. This may result in a long search process time that exceeds the time limitation imposed by design specifications. The lower the number of hash collisions is created by the hash algorithm the better the performance becomes. Performance of a hashing algorithm is usually determined by two measurements: the MSL (maximum search length) and ASL (average search length) with the former one indicating the largest number of hash collisions for any single hash value and the latter denoting the average number of hash collisions for all hash values.

Hashing techniques using simple XOR operations have been very popular in applications where timely response is critical due to its relatively small hash process delay. In all the current commonly used XOR-hashing algorithms, when deriving the hash value, key bits are partitioned into rows to be XORed, and each of the hash key bits is usually explicitly XORed only at most once in the hash process, which may limit the amount of potential randomness that can be introduced by the hash process. When a key bit is reused (duplicated) for XORing in generating different hash value bits, there exists a potential that the overall randomness of new hash result may increase. In [1] a theory has been developed in duplicating bits while avoiding induced bit correlation which may easily offset any gained performance through bit duplication. Very significant performance improvement was obtained in this series of techniques by employing a novel single-row bit duplica- tion process to avoid bit nullification or downgrading problem. An extension from a recent publication in [17], this paper aims to further extend the theory into duplicating more than one rows of key bits. By relaxing the restriction in duplicating only one row of key bits, one is able to XOR more duplicated bits to obtain each hash bit without coming across the bit nullification or downgrading problem. Our simulation results show that a very significant reduction in the amount of hash collision is obtained by the proposed technique compared to the previously proposed duplication techniques.

\section{XOR-Hashing Methodology}

Throughout this paper, the database under discussion is defined as consisting of $M=2^{m}$ entries with each entry having $n$ bits in length. It can also be viewed as having $n M$-bit vectors with each vector consisting of each respective bit from all entries. An example of $n=8$ and $m=3$ is shown in Figure 1(a). The target

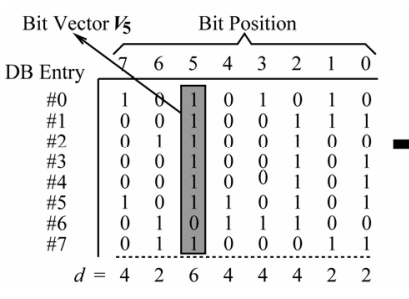

(a)

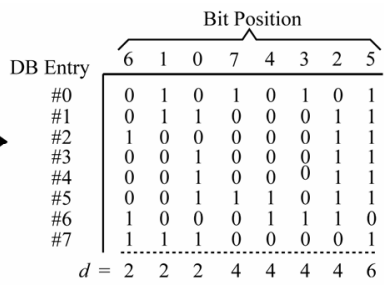

(b)
Figure 1. (a) Calculation of $\boldsymbol{d}$ values (b) sorted database by d value.

hashing process is to hash each of the $n$-bit entries (an IP address or part of it in this application) into an $m$-bit hash value. These hash values need to be distributed as evenly as possible so as to minimize the eventual search time.

XOR operator has been widely used for hashing and known to be an excellent operator in enhancing randomness in distribution. It also possesses a nice characteristic allowing for analytical performance analysis and thus better algorithm designing. A commonly used hashing technique is to simply hash the $n$-bit key into $m$-bit hash result through a simple process XORing every distinct $\frac{n}{m}$ key bits into a final hash bit. Such a random XORing process (so-called "Group-XOR" in this paper) may not always lead to a desirable outcome. A much more effective hashing approach is proposed in [18] by preprocessing (and sorting) the database according to a parameter, the $d$ value, that reveals a very useful insight into the degree of uniformity of the database. The $d$ value of a bit vector is the absolute difference between the number of 0's and 1's in it (as shown in Figure 1(a)). Translated to effect of hashing, in the final $m$-bit hash result, a bit of $d=0$ gives an even hashing distribution (i.e. evenly divided) among the entire address space allowing other bits to hash to it; while a bit of $d=M$ will limit the hashing to only one half of the hash space. Intuitively, using the bits with smaller $d$ values for hashing would lead to a probabilistically better hash distribution, i.e. less potential conflict in the final mapping. Ideally, if one can identify (or through a combination to obtain) $m$ bits with all their $d$ values equal to 0 , it should lead to the best potential performance, assuming no correlation among the bit vectors. This leads us to employing a simple pre-processing step in re-arranging the $n$ bit vectors according to their $d$ values sorted into a non-decreasing order as shown in Figure 1(b). This sorted sequence then gives us an "order of significance" according to which each bit should be utilized.

A XOR-hashing algorithm based on the principle of $d$ value is presented in [19]. This algorithm, the $d$-IOX ( $d$ value in-order XOR folding), involves the aforemen- 
tioned preprocessing/sorting step before applying the simple in-order folding XOR hashing. Figure 2 shows the folding process in the $d$-IOX algorithm, with each of the $H_{i}$ 's referring to a hashing function in deriving a hash value bit. The $d$-IOX proves to be much better than the simple random Group-XOR approach by registering an improvement in ASL and MSL up to $30 \%$ in randomly generated database and up to $80 \%$ in real IP database. Reasons behind such a significant improvement have been clearly explained in [19], mainly due to the nature in XORing bits with very different $d$ values in order to maximize the reduction in $d$ values in XORing.

The new technique proposed in this paper will be based on the result of preprocessing by exploiting the "order of significance" among the key bits for bit reuse/ duplication, and the performance will be compared to the $d$-IOX as the base non-duplication one due to its superior performance over all other well-known XOR-hashing techniques.

\section{Bit-Duplication XOR Hashing}

For the sake of completeness, a summary of the bitduplication theory presented in [1] is given here.

Note that when there is no bit duplication under standard XOR hashing, no bits are shared in XORing to lead to different hash value bits. That is, each hash value bit comes from XORing a distinct set of hash key bits. If one intends to reuse some key bits for XORing, then the overall effectiveness may be compromised due to the sharing. Here a notion is introduced to illustrate the induced effect from bit duplication. In obtaining two hash key bits, when there exist common bits between the two sets of hash key bits for their XORing, an Induced Duplication Correlation (IDC) arises between the two hash value bits. The reason that this correlation is regarded as "induced" because it is created through the artificial bit-sharing from duplication, in contrast to the inherent correlation that may already exist in the hash key bits. Figure $\mathbf{3}$ gives a simple illustration for IDC, where each of the letters (from A to F) denotes a distinct hash key bit.

The figure on the left shows that a hash value bit is obtained by XORing two distinct hash key bits, while the figure on the right shows that through duplication IDC occurs when every pair of hash value bits have some common bits being XORed. When more bits are duplicated for XORing, higher IDC tends to ensue. With the introduction of IDC the $d$ value obtained for each hash value bit loses some of its meaning. That is, while randomness in the bit-wise distribution ( $d$ value) for each bit may be increased due to more bits being XORed, the overall randomness across the $m$ hash value bits may

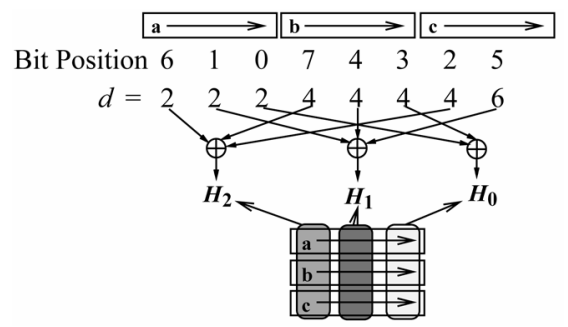

Figure 2. In-order XOR (d-IOX) hash algorithm.

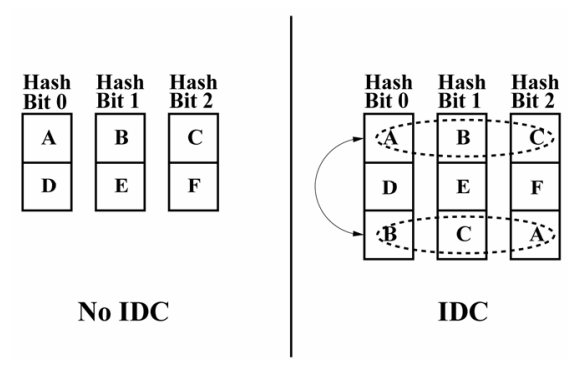

Figure 3. Induced duplication correlation (IDC).

actually decrease due to the IDC. In order to reach the best hash performance, one will need to duplicate effectively while making an effort to reduce the effect of IDC.

In [1], a simple "cycle duplication" approach is proposed to ensure minimal bit correlation through the duplication process, in which key bits are shared between two groups of source bits to be XORed. One typical problem is the "nullification" problem where the same bit is duplicated to be XORed with itself in producing a hash bit, which results in a loss of one additional potential bit for randomness. The other problem is, while performing the cycle duplication process on the same row as shown in Figure 4 where the first row is duplicated twice in order to further increase the randomness. With this, each pair of hash bits will have two key bits shared in their XORing (e.g. bits 0 and 1 sharing $A$ and $D)$, thus leading to a "downgrading" problem, or simply a higher degree of IDC. Note that, in our discussion here, the same segment (the one with the smallest $d$ values) is used for multiple duplication due to its low $d$ values. Using another different segment (a segment of bits with larger $d$ values) for the additional duplication has shown a far less potential for performance improvement due to an analysis result from [18] showing that XORing with bits of larger $d$ values may even degrade the performance. Therefore, in order to avoid any kind of downgrading, one has to first decide if there are sufficient number of bits for further duplication. In order to duplicate $X$ times without the downgrading problem, [1] shows that the minimal $m$ required is

$$
m \geq X \cdot(X+1)+1
$$




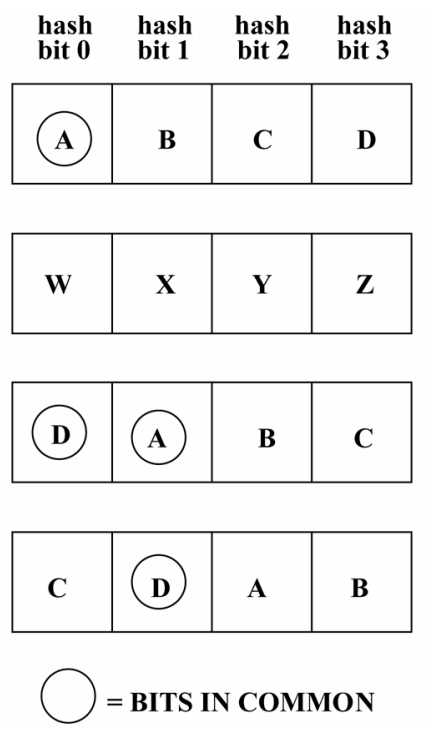

Figure 4. An example of downgrading from additional duplication.

$$
X \leq\left\lfloor\frac{\sqrt{4 m-3}-1}{2}\right\rfloor
$$

In the preceding example shown in Figure 4, where $m=4$ and $X=2$ (two duplications are to be attempted), obviously, the condition in Equation (1) is violated - minimum value of $m$ for two times of duplication is 7 .

The problem can be translated into a problem of graphics for easier visualization and processing. Borrowing from the notations used in [1], let the set of the $m$ bit position indices be denoted $S=\{0,1,2, \cdots, m-1\}$, and these bits are to be duplicated $X$ times such that $X$ satisfies the condition in Equation (1). For the sake of simplicity without losing generality, assume that each of the $X$ duplicated sequences of the $m$ bits are to be rotated starting from a particular bit position to avoid the two problems. We can simply focus on the bit 0 position of each of the strings to analyze the whole pattern. That is, the bit 0 position of the original string is at position 0 . Let the position of bit 0 of each of the $X+1$ strings be denoted as $s_{j}$ where $0 \leq j \leq X$. Figure 5 shows an illustration for a case with $X=3$ and $m=13$, with 13 bit positions on a circle. In this case, the four starting locations are $s_{0}=0, s_{1}=1, s_{2}=3$ and $s_{3}=9$. With this notation, one can easily show that, in order to avoid any nullification problem, the following condition has to hold:

$$
s_{i} \neq s_{j}, \forall i, j, 0 \leq i, j \leq m \text {, and } i \neq j
$$

which guarantees that no bit position has two identical bits to be XORed. In order to avoid any sharing of multiple bits (i.e. the downgrading problem), the follow-
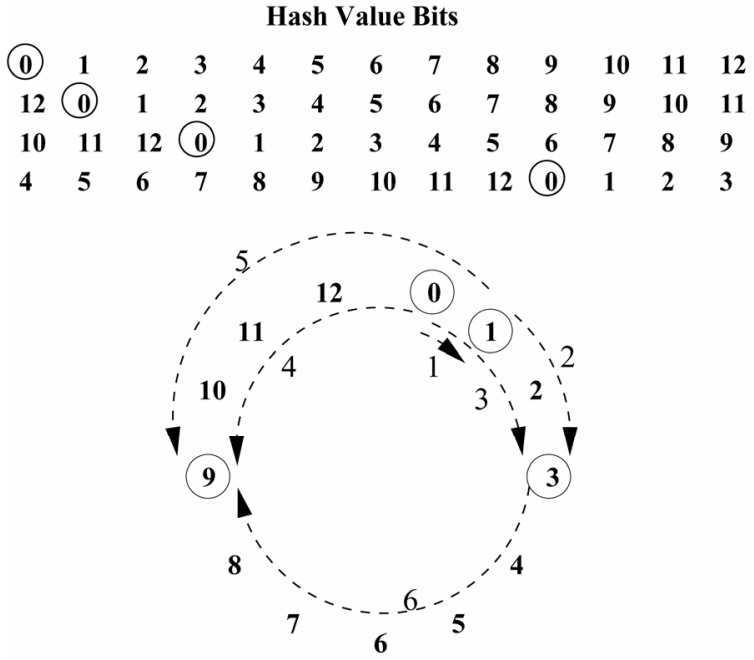

Figure 5. Three-time duplication with $m=13$ using cycle duplication with one bit in common.

ing condition has to be satisfied:

$D_{i j} \neq D_{k l}, \forall i, j, k, l, 0 \leq i, j, k, l \leq m$, and $(i, j) \neq(k, l)$.

where $D_{i j}$ denotes the "shorter" distance from position $s_{i}$ to position $s_{j}$; that is,

$$
D_{i j}=\min \left(\left(s_{i}-s_{j}\right) \bmod m,\left(s_{j}-s_{i}\right) \bmod m\right)
$$

For example, between $s_{1}=1$ and $s_{3}=9$, their distance is

$D_{13}=\min ((1-9) \bmod 13,(9-1) \bmod 13)=\min (5,8)=5$.

Essentially, this condition guarantees that the no two positions can share more than one bit in common. Note that there are a total of $C_{2}^{X+1}$ such distance values among the $X+1$ starting positions (e.g. the six different distance values, $\{1,2,3,4,5,6\}$, in Figure 5), and this number should be exactly the same as the number of all possible "shorter" distance values available from $m$ positions along a circle, which is $\left\lfloor\frac{m}{2}\right\rfloor$, when $m$ is set to be the exact minimum that satisfies Equation (1). Thus, one should be able to prove that

$$
C_{2}^{X+1}=\left\lfloor\frac{m}{2}\right\rfloor
$$

which can be easily derived from

$$
\left\lfloor\frac{m}{2}\right\rfloor=\left\lfloor\frac{X(X+1)+1}{2}\right\rfloor=\frac{X(X+1)}{2}=C_{2}^{X+1} .
$$

\section{Multi-Row "Each-Row-Once” Duplication}

All duplication techniques discussed so far have been the 
"Uni-row duplication" ones, i.e. only the first row is duplicated, potentially multiple times. In order to avoid the problems of nullification and downgrading, the number of times that this row is allowed to be duplicated is limited by $O(\sqrt{m})$. While the benefit in duplicating only "the best row" clearly demonstrates itself, the potential in duplicating more times using other rows cannot be simply ignored. One can easily see that, based on the observation made so far, if the first row is not "fully" duplicated, then the "shorter" distance value slots thus freed up may be used for duplicating other rows, potentially more times.

Based on the theory developed in the previous section, one can easily derive that, if each row is only duplicated once, the total of number of rows (and thus total of times) that can be duplicated without causing the nullification or downgrading problem can be significantly greater than $\left\lfloor\frac{\sqrt{4 m-3}-1}{2}\right\rfloor$. Assuming that the bit rows are indexed as $r_{i}, 1 \leq i \leq\left\lceil\frac{n}{m}\right\rceil$, using the same circular fashion for duplication, one can show that, if $\forall i, 1 \leq i \leq\left\lfloor\frac{m-1}{2}\right\rfloor$, row $i$ is duplicated exactly once by rotating its bits by $i$ bit positions, then the maximum number of duplications can be achieved without any of the aforementioned problems. That is, let $Y$ denote the number of rows thus duplicated, and

$$
Y \leq\left\lfloor\frac{m-1}{2}\right\rfloor
$$

This is demonstrated by the example shown in Figure 6, where each of the three rows, $r_{1}, r_{2}$, and $r_{3}$, is cycle-duplicated once by rotating each of its bits once, twice, or thrice, respectively. If one additional row $r_{4}$ is allowed to duplicated, as shown in Figure 7, the problem of downgrading arises leading to two shared bits in some pairs of two columns.

For examples, bit postions 1 and 5 now share key bits $T$ and $\beta$, and bit positions 0 and 3 share key bits $O$ and $\delta$.

Note that the number of duplications allowed is also limited by the number of rows. Let $X$ be the maximum number of duplications allowed using "uni-row", and $Y$ be the maximum number of duplications allowed using "multi-row each-row-once", Table 1 lists the comparison between these two numbers under different $m$ values. Note that $Y$ denotes the number of allowable duplications when $n$ is unlimited which imposes no restriction on the number of rows available. A more practical illustration is given here with $Y_{32}$ and $Y_{64}$, each denoting the number when $n$ is set to 32 and 64 respectively.

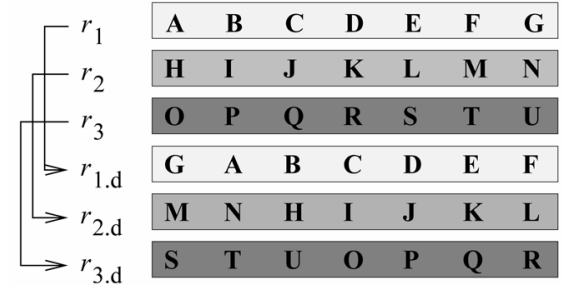

Figure 6. "Each-row-once" duplication with $m=7$ using cycle duplication.

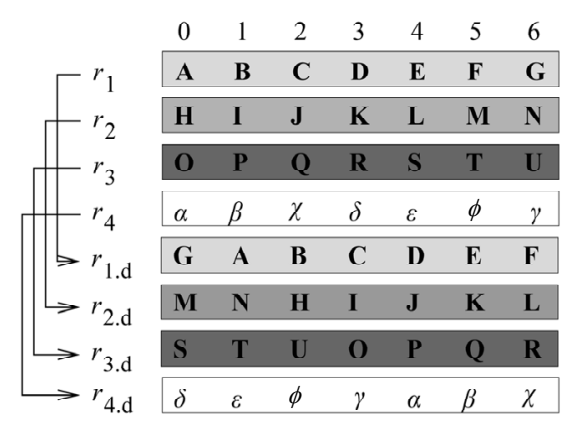

Figure 7. "Each-row-once" over-duplication with $\boldsymbol{m}=7$ using cycle duplication.

Table 1. Maximum number of duplications allowed for a given $\boldsymbol{m}$.

\begin{tabular}{ccccc}
\hline$m$ & $X$ & $Y$ & $Y_{<32>}$ & $Y_{<64>}$ \\
\hline 5 & 2 & 2 & 2 & 2 \\
6 & 2 & 2 & 2 & 2 \\
7 & 2 & 3 & 3 & 3 \\
8 & 2 & 3 & 3 & 3 \\
9 & 2 & 4 & $4^{*}$ & 4 \\
10 & 2 & 4 & $4^{*}$ & 4 \\
11 & 2 & 5 & $3^{*}$ & 5 \\
12 & 2 & 5 & $3^{*}$ & 5 \\
13 & 3 & 6 & $3^{*}$ & $5^{*}$ \\
14 & 3 & 6 & $3^{*}$ & $5^{*}$ \\
15 & 3 & 7 & $3^{*}$ & $5^{*}$ \\
\hline
\end{tabular}

Also, whenever $n$ is not an integral multiple of $m$, the last row is with only a partial length, which is reflected in the table with an *. That is, the maximum duplication times for $Y_{n}$ when there are $n$ bits is

$$
Y_{\langle n\rangle}=\min \left(\left\lfloor\frac{m-1}{2}\right\rfloor,\left\lceil\frac{n}{m}\right\rceil\right)
$$

For example, for $m=15$ and $n=64$, under uni-row duplication scheme, the row for duplication can only be duplicated at most 3 times, while, under this multi-row 
approach, all 5 rows can be duplicated, each row once. Whether or not such a new approach brings additional benefit remains to be decided. For the purpose of discussion, let $X=\left(X_{1}, X_{2}, \cdots, X_{\left[\frac{n}{m}\right]}\right)$ represent the pattern of duplication for all rows, where $X_{i}, 1 \leq i \leq\left\lceil\frac{n}{m}\right\rceil$ denotes the number of times row $i$ is duplicated. Thus, under uni-row duplication, $(3,0,0,0,0)$ is the most duplication allowed; while under multi-row duplication just discussed, $(1,1,1,1,1)$ becomes the pattern for duplication.

\section{Hybrid Duplication}

Note that, as aforementioned, duplicating rows with higher $d$ values tend to bring limited benefits and sometimes can even be detrimental. In order to maximize the benefit from duplication, one may have to use a hybrid approach in duplicating several rows, by duplicating different rows different number of times. Let $X_{i}$ denote the number of times row $i$ is to be duplicated, in order for no nullification or downgrading to happen, the following condition has to be satisfied.

$$
\sum_{i}^{\left\lceil\frac{n}{m}\right.} \frac{X_{i}\left(X_{i}+1\right)}{2} \leq\left\lfloor\frac{m-1}{2}\right\rfloor
$$

where $r$ is the total number of rows under duplication. Note that this is a necessary condition but not a sufficient condition since a given pattern satisfying this condition may not be feasible. A simple example is when $m=14$, a satisfying pattern of $(2,2)$ cannot be constructed. This again follows the same reasoning in proving the uni-row and multi-row duplication. Similarly, under $m=15$ and $n=64$, some of "maximally" allowed duplication patterns are listed in the following: $(3,1,0,0,0)$, $(2,1,1,1,1),(2,2,1,0,0)$, and all permutations of each of the patterns, such as $(0,1,0,3,0)$ and $(1,2,0,1,2)$. For example, in the case of $(2,2,1,0,0)$, as shown in Figure 8, first row is duplicated two times with each of the three starting bit positions being 0,1 and 3 (indicated in circles), the second row is duplicated two times with the starting positions being 0,4 and 9 (indicated in boxes), and the third row duplicated once with the two starting positions being 0 and 7 (indicated in triangles). Note that, the condition for avoiding nullification problem still needs to be satisfied for each of the three duplicated rows, independently; that is, all three rows duplicated are allowed to share the starting bit position 0 . For the condition in avoiding downgrading problem (Equation (3)), again, it has to be satisfied within each

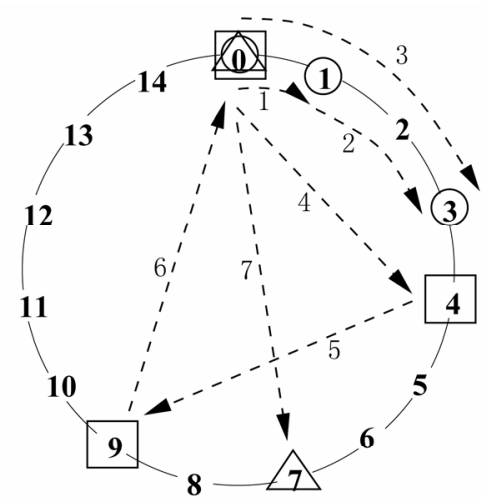

: starting bit positions of first row and its duplications : starting bit positions of second row and its duplications : starting bit positions of third row and its duplications

Figure 8. An example of hybrid duplication with $(2,2,1,0,0)$ on $m=15$.

set of duplicated rows; that is, for the first row, the three distance values between every pair of two starting positions are 1 (between 0 and 1), 2 (between 1 and 3) and 3 (between ( 0 and 3 ); for the second row the three distance values are 4 (between 0 and 4), 5 (between 4 and 9) and 6 (between 9 and 0); for the third row the only distance value is 7 (between 0 and 7). With this, all available distance values (from 1 to 7 ) are taken, which represents the maximally allowed duplication situation. The complete duplication pattern is shown in Figure 9.

\section{Simulation Results}

Simulation runs are performed on randomly generated data sets and real IP data sets to demonstrate the performance improvement of the minimal IDC dupli- cation XOR hash technique over other techniques with no duplication. The Group-XOR algorithm which XORs groups of random key bits is the general base of our comparison, while the $d$-IOX [19] and the IDC technique from [1] aforementioned will serve as the reference.

\subsection{Randomly Generated Data Sets}

We first use a data set randomly generated such that the $d$ value for each bit position is uniformly distributed. Performance comparison among the three techniques are in terms of MSL and ASL by taking an average of results from 1000 runs. In order to disengage potential effect

\footnotetext{
1“Partial-row" duplication poses an additional relaxation on the constraint from Equation (1) since the last partial row is not fully cycled around the whole $m$-bit section thus leading to less restriction on the downgrading. This issue will be disregarded in this paper due to its minimal effect on the performance from duplicating the last row which has the largest $d$ values.
} 


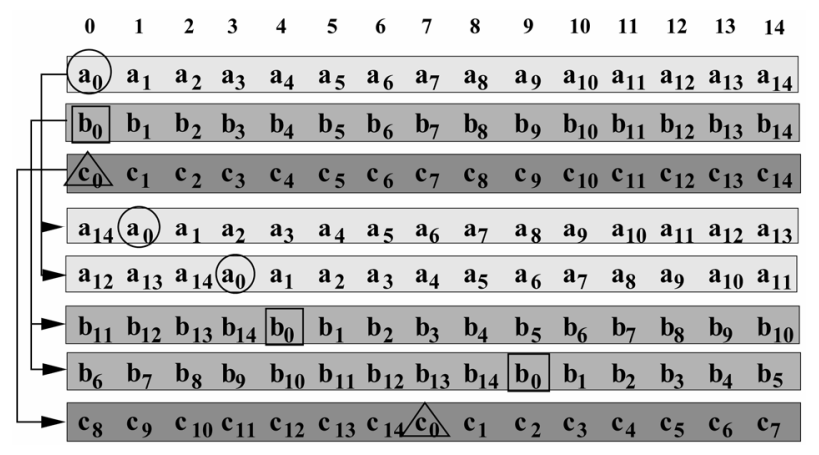

Figure 9. Hybrid Duplication with $(2,2,1,0,0)$ on $m=15$.

from any uncertain factors, no partial rows are considered; that is, $n$ is set to be an integral multiple of $m$.

We first compare the effect of uni-row duplication approaches in duplicating different rows. Figure 10 shows the results comparing all possible uni-row duplication patterns on various of combinations of $(n, m)$ when $n$ is set to $2 m-(32,16),(30,15)$ and $(28,14)$.

Figure 11 shows the results when $n$ is set to $3 m$ $(30,10),(27,9),(24,8)$. Throughout these different combinations, when one row is selected for duplication, the more times it is duplicated, the higher the performance it delivers. Comparing performance from duplicating different rows, our aforementioned conjecture is clearly verified here that duplicating the row with the smallest $d$ values (the first row) does lead to the most benefit while duplicating the row with the largest $d$ values produces the least benefit.

On the cases where $n=3 m$, the uni-row duplication shows a somewhat different result than the $n=2 m$ cases. Duplicating the best row (the first row) again shows the best potential, while duplicating each of the non-best rows (the second or the third row), although it still shows continuously improved performance when more duplications are applied, its best achievable performance (from $(0,2,0)$ or $(0,0,2)$ ) cannot closely match the performance from $(2,0,0)$. From this result, had the second row been allowed to duplicate a few more times, it might have had a chance to match the first-row duplication, but the maximal number of times that can be applied without causing any nullification or downgrading problem is restricted to 2 for each of the uni-row duplications under $m \leq 10$ for our simulations. Duplicating the third row does not inspire as much as duplicating other rows, which can be easily explained by the fact that the high $d$ values in the third row inherently limit its potential in duplication. Under uni-row duplication, the best duplication is from the maximally duplicated patterns using the first row.

Using the each-row-once duplication approach, one may be able to duplicate the most number of times, but
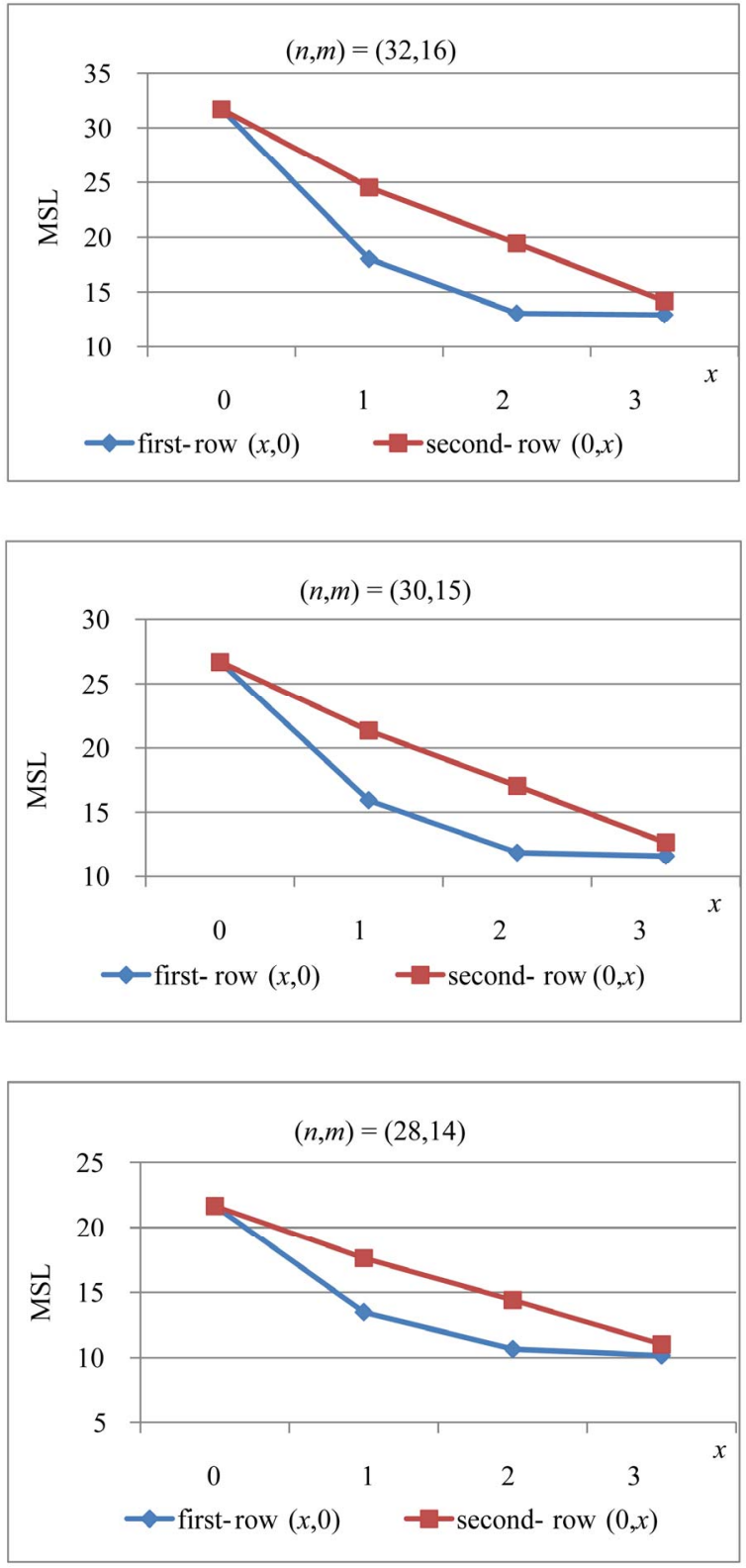

Figure 10. Simulation results for uni-row duplications for $\boldsymbol{n}$ $=2 m$.

the benefit may be offset by duplicating the rows with larger $d$ values, and the fact that $m$ is not large enough to support maximal number of rows for duplication also limits the potential of this approach.

Simulation runs on hybrid duplication deliver the most intriguing results. Figure 12 and Figure 13 show the comparison results for $n=2 m$ and $n=3 m$, respectively. In the cases where $n=2 m$, some of hybrid duplication patterns easily outperform the best uni-row duplication approach. For example, in the case of $(n, m)=(32,16)$, the the patterns of $(1,3),(2,1),(2,2)$ and $(3,1)$ all exceed the performance of $(3,0)$ by a 

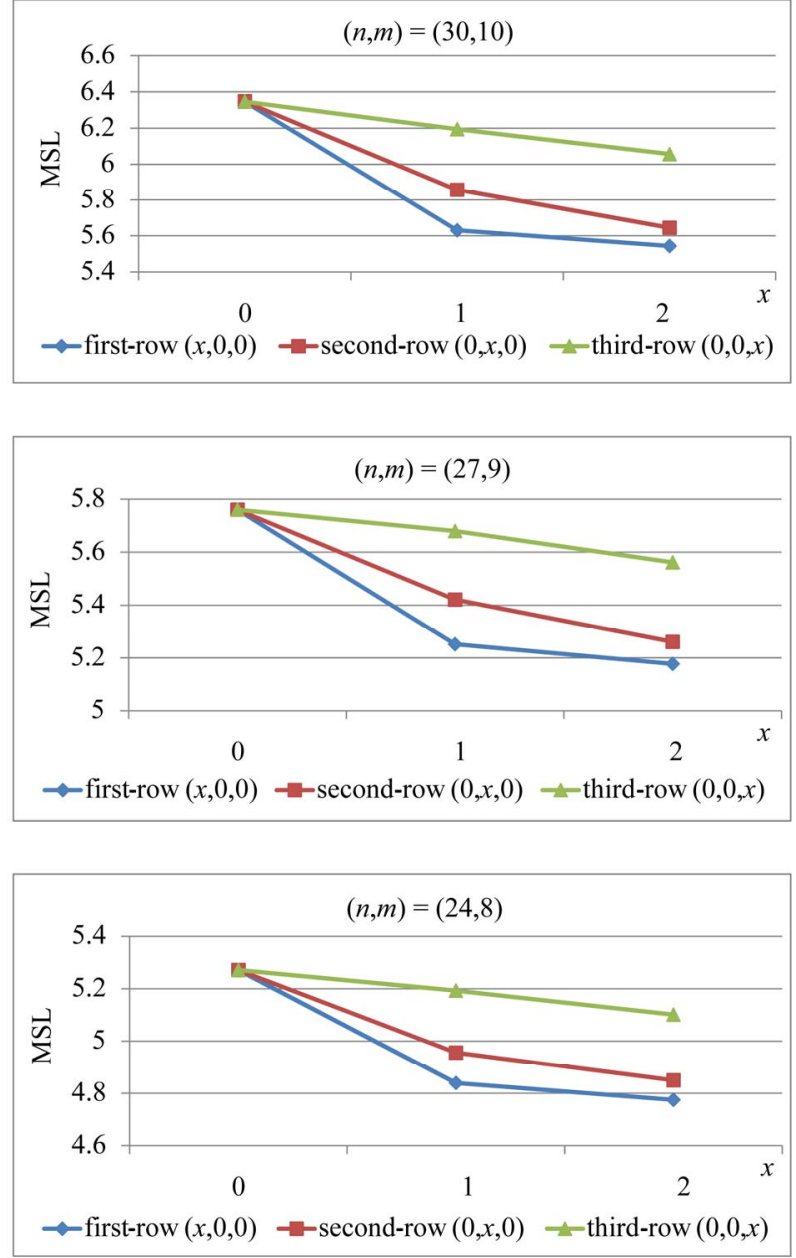

Figure 11. Simulation results for uni-row duplications for $n$ $=3 m$.

significant margin, up to an additional $25 \%$ of improvement. Similarly, an additional $22 \%$ and $18 \%$ of improvement is obtained in the case of $(n, m)=(30,15)$ and $(n, m)=(28,14)$, respectively. This important observation reveals that maximally duplicating the best row, $(3,0)$ in this case, may not be the best approach; instead, duplicating the best row fewer than the maximally allowed times coupled with duplicating the second row (e.g. $(2,1)$ or $(2,2)$ or even $(1,2))$ actually delivers better results. This can be explained by looking into the performance trend shown in Figures $\mathbf{1 0}$ and $\mathbf{1 1}$ where duplicating a row with smaller $d$ values tends to reach it best potential earlier in terms of the numbers of duplication applied. For example, $(3,0)$ does not pose a significant gain over $(2,0)$, while duplicating the second row each additional time obviously provides more benefit. In the cases of $n=3 m$, hybrid patterns produce even more interesting results. First, the pattern of $(1,1,1)$ by duplicating the third row on top of $(1,1,0)$ actually leads to a degraded performance, which again
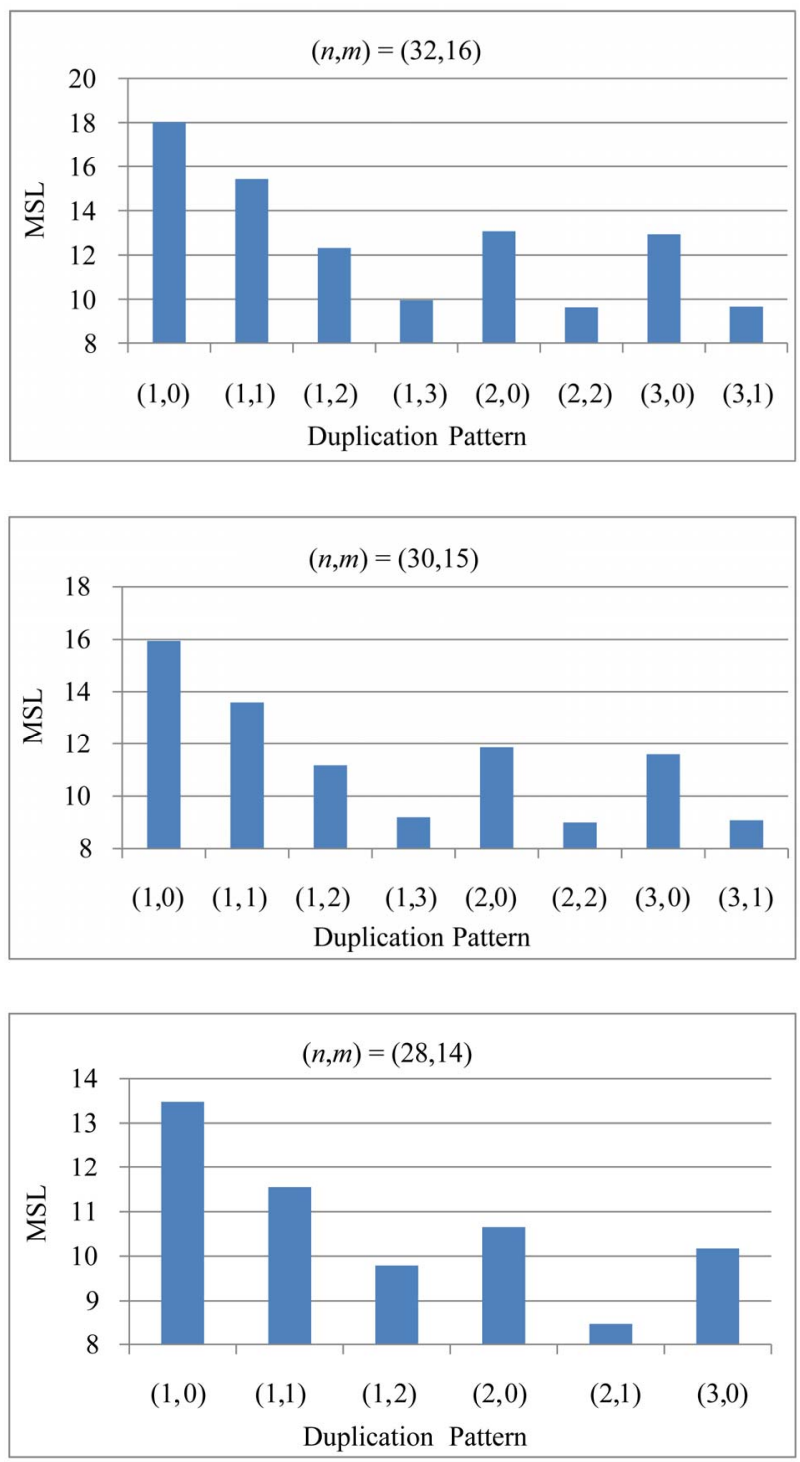

Figure 12. Simulation results for hybrid duplications for $n$ $=2 m$.

can be explained by the large $d$ values in the third row. In general, duplicating the first row and second row wherever is feasible normally leads to a gain in performance. In $(n, m)=(30,10)$ and $(n, m)=(27,9),(2,1,0)$ produces the best performance, closely followed by that of $(1,2,0)$. In $(n, m)=(24,8)$, due to the limitation of $m$, neither $(2,1,0)$ nor $(1,2,0)$ is feasible, and $(2,0,0)$ easily leads the pack followed by $(1,1,0)$.

\subsection{Downgrading Problem}

We further verify our claim in the downgrading problem using a series of simulation runs. Instead of using the properly selected starting bit(s) in rotation for duplication, a straightforward "one-bit shift" rotation is employed. 

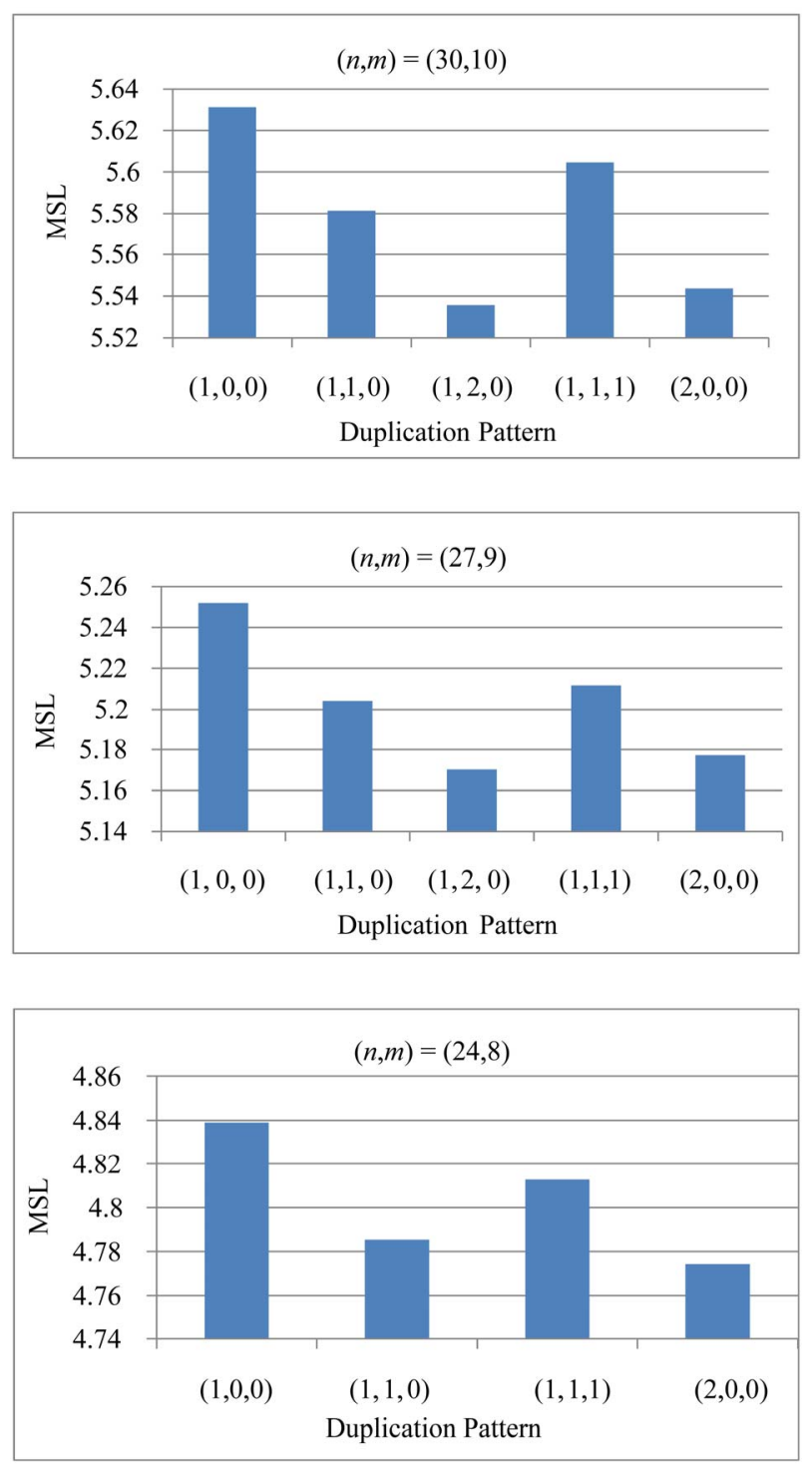

Figure 13. Simulation results for hybrid duplications for $n$ $=3 m$.

Figure 14 illustrates the comparison between the two, with $m=16$ and $n=32 .\left(X_{0}, 0\right)$ denotes the standard duplication patterns using the proposed approaches, while $\left(X_{0}^{*}, 0\right)$ denotes the simple "one-bit shift" patterns. The results clearly show that, when duplicating the first row twice, the downgrading problem incurs a loss of $11 \%$ in performance, while it reduces the gain by $6 \%$ when duplicating three times. Note that, properly duplicating twice (shows as $(2,0)$ ) even outperforms the three-time duplication with a downgrading problem (shown as $\left.\left(3^{*}, 0\right)\right)$.

\subsection{IP Data Simulation}

Simulation is also performed on a collection of real IP

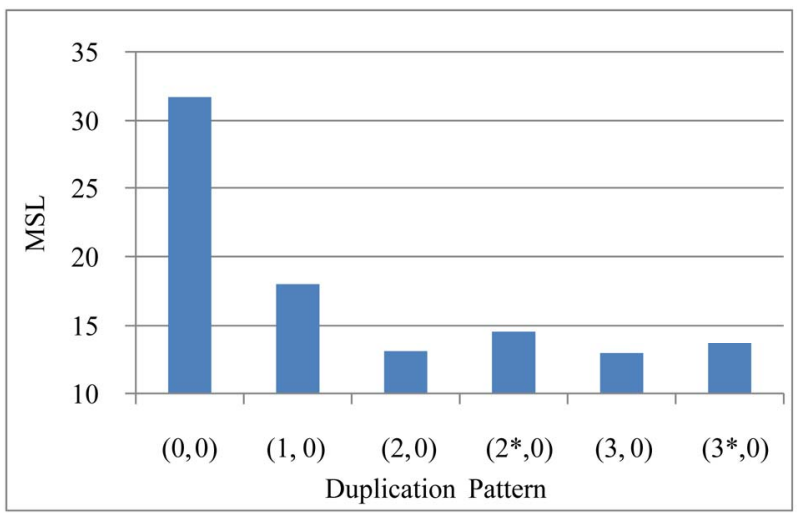

Figure 14. Simulation results illustrating the effect of downgrading in duplication.

addresses gathered from three different sources:

- General IP Traffic

- $\mathrm{Ad} / \mathrm{Spam}$ IP

- P2P IP

The General IP traffic addresses are collected from packets entering a local network router in a duration of a few hours, while the Ad/Spam and P2P IP addresses are gathered from the IP filtering open source software project PeerGuardian [20]. For the simulation on these data sets, $2^{m}$ distinct IP addresses were randomly taken from the trace and then used as a database to perform the hashing. Results are obtained by averaging those from 1000 runs. Figures 15, 16 and 17 display the simulation results comparing among different duplication schemes under various values of $m$ when $n$ is set to equal to $2 m$. Results from general IP address database exhibit a trend similar to what is obtained from the random database. In general, the more times a row is duplicated, the better the performance becomes, which applies to each of the two rows. Hybrid duplication patterns usually lead to better performance, which is clearly demonstrated in the cases of $m=15$ and $m=14$ where $(2,2)$ outperforms all others in the former case and $(1,2)$ and $(2,1)$ both excel in the latter case. In the case of $m=16,(0,3)$ barely beats out a group of hybrid patterns, including $(3,1),(1,2),(2,2)$ and $(1,3)$.

Results from Ad-Spam IP address database (Figure 16) show a very different scenario. When increasing the number of duplication on the first row, results in general improves; duplication of the second row leads to a very surprising behavior. Duplicating the second row once, $(0,1)$, leads to a dramatically degraded performance by a margin from $6 \%$ in $m=14$ to $33 \%$ in $m=16$. Further duplicating the second row with $(0,2)$ and $(0,3)$ does improve the performance from $(0,1)$ but are still worse than the non-duplication case. This behavior can be attributed to the following reasons. The $d$ value distribution of this database after sorting the key bits accor- 

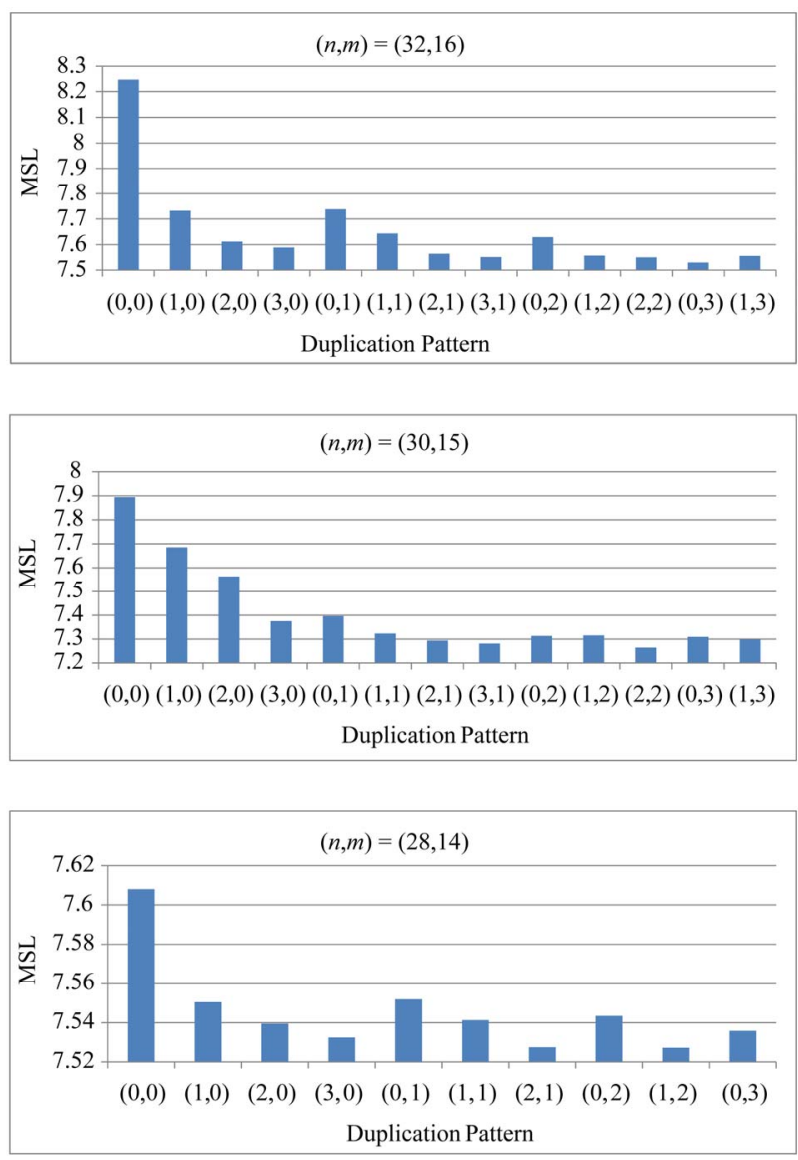

Figure 15. Simulation results for general IP addresses for (a) $n=2 m$.

ding to the $d$ value is very different from the general IP one and especially from the randomly generated one. The $d$ values of most key bits of this database are already small compared to the other databases, which leads to a better result compared to other database when no duplication is used (case $(0,0))$. According to our previous theoretical analysis, it is harder to further reduce the $d$ value of a bit already with a small $d$ value by XORing it with another bit with a $d$ value not significantly larger [18]. When duplicating the second row which has $d$ values not significantly larger than the first row's, the resulted $d$ values after XORing tend to increase. Note that the degree of performance degradation from duplicating the second row lessens as $m$ becomes smaller. For this database, the best duplication pattern is clearly dominated by the duplication of only the first row $(0,3)$ usually outperforms all others.

Simulation from P2P IP address database shows somewhat similar results (Figure 17) to those from Ad-Spam IP database, but the degree of performance degradation from duplicating the second row is not as severe. In the case of $m=14$, such a duplication $((0,1))$
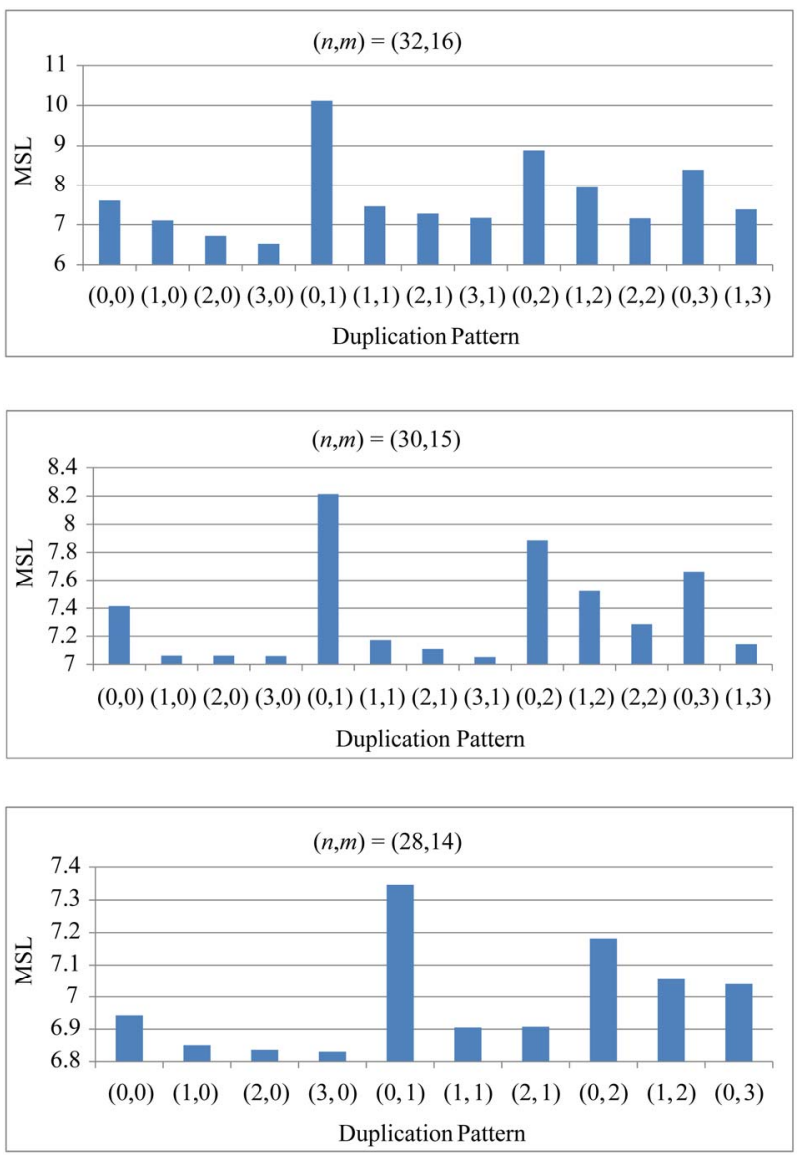

Figure 16. Simulation results for ad-spam IP addresses for $n=2 m$.

actually leads to a $5 \%$ improvement in performance, and it is furthered improved when duplicated more. The best performance is usually achieved with a hybrid pattern, $(2,1)$ for $m=14,(2,2)$ for $m=15$, and $(3,0)$ for $m=16$.

\section{Conclusions}

This paper further extends previously proposed hash design methodology to allow for more performance improvement. This new methodology provides an extra degree of design flexibility and points out a direction for future research, especially for cases with large number of hash key bits. By duplicating and reusing hash key bits in a more comprehensive manner, our technique further enhances the randomness from the best known XORhashing techniques. There still exist many potential extensions along this line of research. This paper only approaches bit duplication in a cyclic pattern, while a mixture of different patterns may provide even more benefit. In addition, this paper examined only induced correlation from the duplication without considering the 

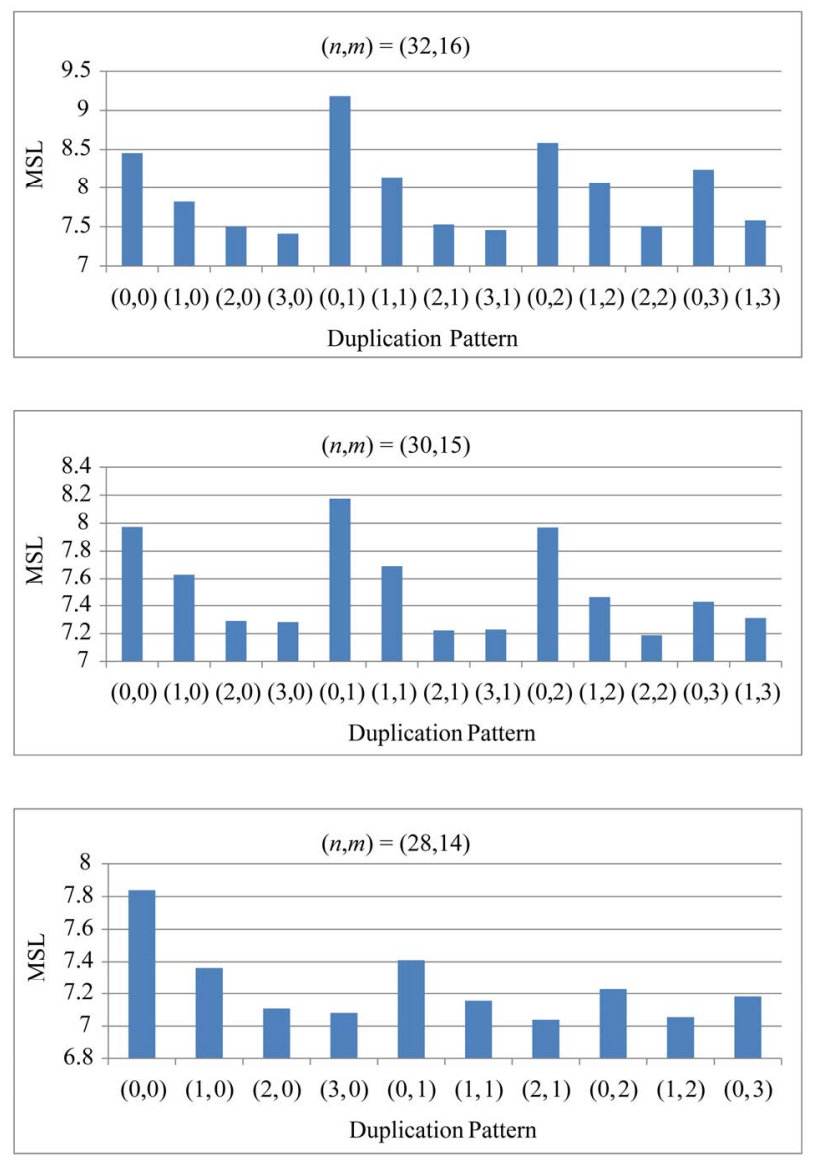

Figure 17. Simulation results for P2P IP addresses for $n=$ $2 m$.

inherent correlation already existing in the target database, which may have a very significant impact on the design of hash algorithms. By providing initial groundwork for duplication in hashing, this paper has pointed out the potential areas to improve hashing algorithms and new ways to exploit specific characteristics of the target database.

\section{References}

[1] C. Martinez and W.-M. Lin, "Advanced Hash Algorithms with Key Bits Duplication for IP Address Lookup," The 5th International Conference on Networking and Services, Valencia, 20-25 April 2009, pp. 137-142.

[2] P. Newman, G. Minshall, T. Lyon and L. Hutson, "IP Switching and Gigabit Routers," IEEE Communications Magazine, Vol. 35, No. 1, 1997, pp. 64-69. doi: $10.1109 / 35.568212$

[3] O. Amble and D. E. Knuth, "Ordered Hash Tables," Computer Journal, Vol. 17, No. 2, 1974, pp. 135-142. doi:10.1093/comjnl/17.2.135

[4] M. A. Ruiz-Sanchez, E. W. Biersack and W. Dabbous, "Survey and Taxonomy of IP Address Lookup Algo- rithms," IEEE Network, Vol. 15, No. 2, 2001, pp. 8-23. doi:10.1109/65.912716

[5] R. Jain, "A Comparison of Hashing Schemes for Address Lookup in Computer Networks," IEEE Transactions on Communications, Vol. 40, No. 10, October 1992, pp. 15701573. doi: $10.1109 / 26.168785$

[6] A. Moestedt and P. Sjodin, "IP Address Lookup in Hardware for High-Speed Routing," Proceedings of IEEE Hot Interconnects 6 Symposium, Stanford, 13-15 August 1998, pp. 31-39.

[7] X. J. Nie, D. J. Wilson, J. Cornet, D. Damm and Y. Q. Zhao, "IP Address Lookup Using A Dynamic Hash Function," Canadian Conference on Electrical and Computer Engineering, Saskatoon, 1-4 May 2005, pp. 1642-1647.

[8] S. Nilsson and G. Karlsson, "IP Address Lookup Using LC-Tries," IEEE Journal on Selected Areas in Communications, Vol. 17, No. 6, June 1999, pp. 1083-1092. doi:10.1109/49.772439

[9] V. Srinivasan and G. Varghese, "Faster IP Lookups Using Controlled Prefix Expansion," Proceedings of the 1998 ACM SIGMETRICS Joint International Conference on Measurement and Modeling of Computer Systems, Madison, 22-26 June 1998, pp. 1-10.

[10] M. Waldvogel, G. Varghese, J. Turner and B. Plattner, "Scalable High Speed IP Routing Lookups," Proceedings of the ACM SIGCOMM'97 Conference on Applications, Technologies, Architectures, and Protocols for Computer Communication, Cannes, 14-18 September 1999, pp. 25 35.

[11] A. Broder and M. Mitzenmacher, "Using Multiple Hash Functions to Improve IP Lookups," 20th Annual Joint Conference of the IEEE Computer and Communications Societies, Anchorage, 22-26 April 2001, pp. 1454-1463.

[12] S.-H. Chung, S. Jean, H. Yoon and J.-W. Cho, "A Fast and Updatable IP Address Lookup Scheme," International Conference on Computer Networks and Mobile Computing, Beijing, 16-19 October 2001, pp. 419-424.

[13] D. Yu, B. Smith and B. Wei, "Forwarding Engine for Fast Routing Lookups and Updates," Global Telecommunications Conference, Rio de Janeireo, 5-9 December 1999, pp.1556-1564.

[14] C. Martinez, D. Pandya and W.-M. Lin, "On Designing Fast Non-Uniformly Distributed IP Address Lookup Hashing Algorithms," IEEE/ACM Transactions on Networking, Vol. 17, No. 6, December 2009, pp. 1916-1925. doi:10.1109/TNET.2009.2014949

[15] D. Pao, C. Liu, L. Yeung and K. S. Chan, "Efficient Hardware Architecture for Fast IP Address Lookup," IEEE Proceedings of Computers and Digital Techniques, Vol. 150, No. 1, 2003, pp. 43-52. doi:10.1049/ip-cdt:20030082

[16] P. A. Yilmaz, A. Belenkiy, N. Uzun, N. Gogate and M. Toy, "A Trie-Based Algorithm for IP Lookup Problem," Global Telecommunications Conference, San Francisco, 27 November - 1 December 2000, pp. 593-598.

[17] R. Tiengtavat and W.-M. Lin, "Advanced Hashing with Hybrid Key Duplication for IP Address Lookup," The 9th 
IEEE International Symposium on Network Computing and Applications, Cambridge, 15-17 July 2010, pp. 261264.

[18] C. Martinez and W.-M. Lin, "Adaptive Hashing Technique for IP Address Lookup in Computer Networks," 14th IEEE International Conference on Networks, Singapore, 13-15 September 2006, pp. 198-203. doi:10.1109/ICON.2006.302586
[19] D. Pandya, C. Martinez, W.-M. Lin and P. Patel, “Advanced Hashing Techniques for Non-Uniformly Distributed IP Address Lookup," 3rd IASTED International Conference on Communications and Computer Networks, Lima, 4-6 October 2006, pp. 46-51.

[20] PeerGuaridan 2, 2007. http://phoenixlabs.org/pg2/ 